\title{
Intelligent process planning and control of DED (directed energy deposition) for rapid manufacturing
}

\author{
Masahiro UEDA*, David CARTER**, Kazuo YAMAZAKI***** and Yasuhiro KAKINUMA* \\ * Department of System Design Engineering, Faculty of Science \& Technology, Keio University \\ 3-14-1 Hiyoshi, Kouhoku-ku, Yokohama-shi, Kanagawa 223-8522, Japan \\ E-mail: m.ueda@keio.jp \\ ** IMS-Mechatronics Laboratory, Department of Mechanical and Aerospace Engineering, University of California Davis \\ One Shields Avenue Davis, CA 95616, USA \\ *** Precision Manufacturing Center, Department of Mechanical Engineering, University of California Berkeley \\ Hearst Avenue Berkeley, CA 94706, USA
}

Received: 25 November 2019; Revised: 21 January 2020; Accepted: 19 February 2020

\begin{abstract}
This research has been conducted to develop computer aided manufacturing software, which can generate NC programs for a five-axis directed energy deposition (DED) type additive manufacturing system with following three focuses: 1. How to maintain consistent formation of a melt pool on the surface of the workpiece. 2. How to generate three-dimensional deposition path efficiently when a 3D part model is given. 3. How to control process parameters during additive operation. For focus 1 , the work orientation has been controlled such that the surface to be deposited can remain perpendicular to the nozzle which is fixed to gravity direction and minimize distortion of the laser spot and powder distortion on the workpiece surface. For focus 2, a unique path generation method has been proposed based on the reverse play of a subtractive machining path, which removes the entire part to the null. For focus 3 , preoperative process parameters control has been introduced to achieve the specified volumetric deposition rates along the additive tool path, which is equated with the material removal rates along the subtractive machining tool path. With methods mentioned above, the dedicated CAM system, CAMAM, has been prototyped and its feasibility has been verified experimentally by applying the $\mathrm{NC}$ program generation strategies to deposit several parts.
\end{abstract}

Keywords : Additive manufacturing, Computer aided manufacturing (CAM), Directed energy deposition, Process optimization, Nozzle orientation control

\section{Introduction}

Additive Manufacturing (AM) has evolved over the last three decades as a promising future-oriented manufacturing technology. For metal based AM, there are two layering methods that are most popular selective laser melting (SLM) and directed energy deposition (DED) (Thompson et al., 2016). The main differentiation between the two is SLM adds material and energy sequentially while DED applies both simultaneously. For SLM, metal powder is initially distributed evenly over a base plate in a chamber. After that, the powder is irradiated by a laser that scans the surface and selectively fuses the powder to the base plate. The base plate is subsequently lowered, and a subsequent powder layer is applied. The additional layer of powder is selectively irradiated fusing the metal powder to the previous layer. These processes of coating and fusion of powder with laser energy repeats until the part is expected to be completed (Liu et al., 2016). The process principle of DED differs from that of SLM. As illustrated in Fig.1, in the DED process, a work piece is irradiated by the laser beam coming through the nozzle and a local heating area, so called as a melt pool, is formed on it into which metal powder is simultaneously fed for deposition (Kuriya et al., 2018). It should be noted that the focus of this paper is a powder type DED while DED encompasses also wire type AM. Metal powder is carried by gas and shield gas is also supplied to prevent powder coming into the nozzle as well as protects laser optical lenses from powder contamination. For these purposes, inert gas is generally used and plays also a role in purging the atmosphere locally from the melt pool 


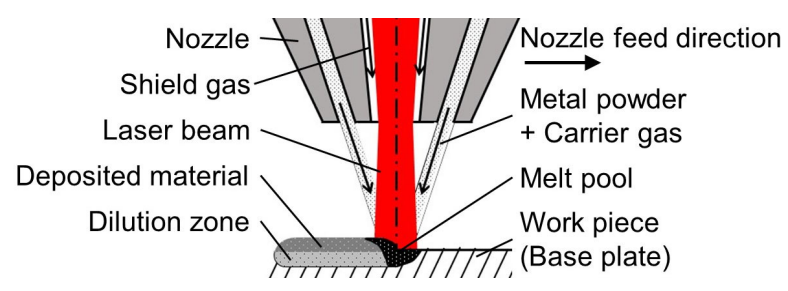

Fig. 1 Process principle of DED using coaxial type nozzle.

area and preventing oxidization. From the viewpoint of this process principle described above, forming a melt pool at the targeted point stable is an important factor for stable process and the geometrical accuracy of the final deposition shape (Xiong et al., 2013). Also, according to the process principle, each type of AM has aspects. For both SLM and DED, slicing method is used to plan the path and build-up parts layer by layer. SLM is limited to build up twodimensionally while it is possible to build in any direction by DED. In addition, SLM usually takes longer to complete the part as compared to DED due to thin slice-stacking method. DED is not required to fill a build chamber with powder so it is more convenient for the fabrication of large objects. Furthermore, DED is suitable not only to build up fully dense parts but also to repair worn out high value components or apply coating to existing parts (Schmidt et al., 2017).

In the study, DED type AM has been chosen for developing the higher degree application technologies of AM for innovative and autonomous manufacturing. For successful operations of a system equipped with DED type AM, there are some attentions or difficulties needs to be emphasized based on above discussion. First, an even melt pool has to remain at the targeted point on the surface of a work piece and metal powder has to be supplied in it constantly during the process. Second, process planning of DED based AM requires the path planning method to specify simultaneous three-dimensional nozzle motion for designed objects. Third, process elements such as nozzle motion, powder supply volume and laser power have to be controlled with respect to the target part geometry. Forth, these process elements need to be modulated synchronously considering different response characteristics. Fifth, the deviation of deposited height from the target value can happen. This causes the change of the distance between the nozzle tip and the deposited surface and result in the change of powder concentration and shielding gas flow at the melt pool, thus the deviation can be worsened. Finally, the thermal state of a deposited part is changed according to the progress of the deposition process and heat accumulation applied by laser beam. In order to realize successful operation of the system, these challenges have to be tackled and solved. In response to the first four requirements, there is a strong need for the dedicated CAM system so as to perform such sophisticated control in automated fashion by the NC program. It is the goal of the CAM system to automatically create a NC program with commands to control AM process. Process planning for DED type AM has been studied thus far (Liou et al., 2010) (Newman et al., 2015). Also, there is some CAM software for DED type AM which is the currently commercially available versions to generate the code for DED (Autodesk Inc., 2018) (CGTech Ltd., 2018). However, not so much CAM software is teaching why and how nozzle motion affects deposition stability from the viewpoint of the process principle to its users. For instance, the nozzle orientation can be set in some CAM software for DED type AM as arbitrarily as CAM software for subtractive machining even though the orientation can change the shape of a melt pool on the deposited surface directly. Therefore, users might have faced to undesired deposition results if they are setting the orientation as they do for subtractive machining process. In addition, the current software is still on a way to a fully developed stage compared to that for the subtractive machining process due to the limitation of tool path creation strategy. For example, users are selecting a path pattern from the specific path patterns already prepared in CAM software according to the target work, when generating a tool path. This could mean that tool paths can be generated only for shapes that conform to the limited patterns. If what a user wants to create does not conform to any pattern supported by the CAM software, it needs to develop a dedicated pattern newly, thus it requires a lot of efforts. Furthermore, CAM software for DED type AM is required to integrate the dynamics of the system and relationships between process parameters and deposition characteristics into process planning. On the other hand, in current CAM software, the deposition parameters remain constant during the entire process even though the required deposition amount can differ according to each tool path point. With respect to the last two requirements, such challenges can be better solved using real time monitoring and feedback control (Ding et al., 2016).

In this research, a dedicated CAM system entitled "CAMAM" (Computer Aided Manufacturing software for DEDtype Additive Manufacturing system) has been proposed and prototyped based on above focuses, and its feasibility was verified in the experimental study. There are three features and corresponding advantages that CAMAM has compared 
with commercial CAM software which has challenges described above. Firstly, optimization of the work orientation using simultaneous five-axis motion control that keeps the deposited surface perpendicular to the nozzle has been proposed by pointing out its effect on stable melt pool formation, thus successful operation for three-dimensional deposition. Secondly, the path of the DED process has been determined by reversing a subtractive machining path which is generated by utilizing existing CAM software for cutting operation and defined to machine the desired object to zero volume. This could enable efficient and versatile AM tool path generation. Thirdly, a method for preoperative control of the fundamental process parameters has been integrated in CAMAM based on the empirical analysis of deposition parameter effects on the deposition geometry. The proposed preoperative control method can solve the challenges of commercial CAM software for DED type AM and contribute the improvement for shape accuracy of deposited parts.

\section{Configuration of DED type AM system}

Figure 2 illustrates the configuration of DED type AM system used in this study. The main machine is designed to use the basic structure of a traditional five-axis milling machine providing rotation in the $\mathrm{A}$ and $\mathrm{C}$ axis directions. The laser head is mounted on the spindle head of the machine which can move $\mathrm{X}, \mathrm{Y}$ and $\mathrm{Z}$ directions. The two rotational axes in addition to the three linear axes allow for the operations to be carried out using simultaneous five-axis motion. The laser beam is delivered by a laser light fiber from the laser unit to the laser head and exits from the nozzle through optics inside of the laser head, then a work piece set on the table is irradiated by the laser beam. It should be pointed out that this configuration is taking on important points as DED type AM system for the following reasons; Frist, this configuration enables to feed powder as well as apply laser always in the direction of gravity. Second, workpiece can be oriented by using $\mathrm{A}$ and $\mathrm{C}$ axis such that the nozzle which direction is fixed in the gravity direction can be remained normal to the surface to be deposited. Laser source type is fiber coupled diode laser which power can be changed from 0 to 2,000W. Metal powder is fed from a powder feeder beside the machine to the laser head by using carrier gas. Shield gas supplied to the laser head is also provided by the same gas source as carrier gas and the amount of gas having the two different purposes can be regulated independently. To operate the system, first, it is required to generate a $\mathrm{NC}$ program from the 3D model of a target object. Such NC program should include not only axis motion information but also how to modulate process parameters throughout the operation. After that, the generated program is run on the NC of the machine and DED operation is finally performed. To conduct this operation flow, in this study, deposition path is determined by performing reverse playback of a subtractive machining path to remove the target geometry to zero volume, and deposition parameters are determined from the removal volume amount which is calculated by the subtractive machining simulation which are described in chapter 4. Figure 3 shows the control block diagram of the system. The process parameters including the powder mass flow rate $\left(=Q_{m}[\mathrm{~g} / \mathrm{min}]\right)$, the laser power $(=P[\mathrm{~W}])$ and the nozzle feed rate $(=F[\mathrm{~mm} / \mathrm{min}])$ can be defined as the process variables in a NC program. Commands to change process variables during deposition are defined using coordinate positions. For example, in the NC program shown in Fig. 3, it is intended to decrease the laser power from $2,000 \mathrm{~W}$ to $1,400 \mathrm{~W}$ when the nozzle starts to move from Y-60 to Y-40.

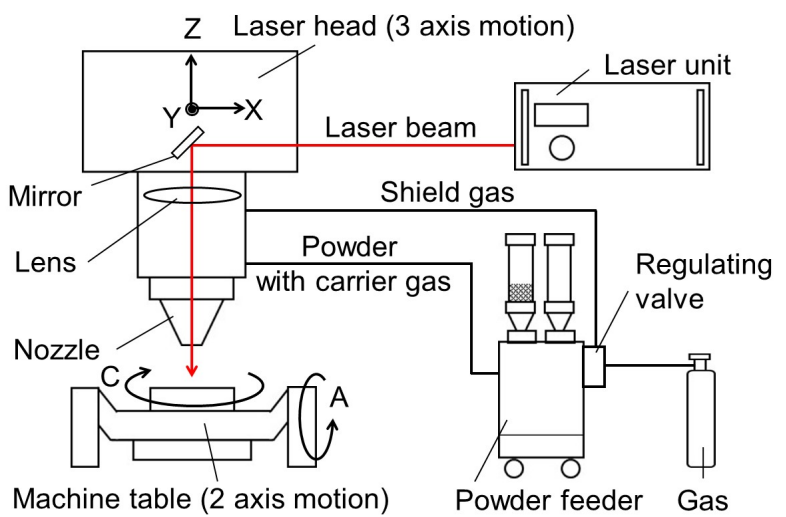

Fig. 2 Configuration of DED type AM system used in this study.

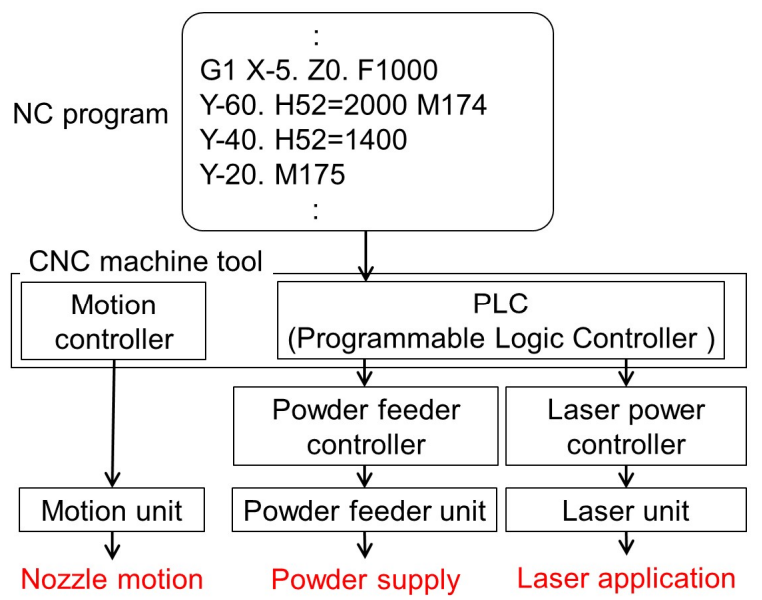

Fig. 3 Control block diagram of DED type AM system. (M174/M175: Laser on/off, H52: Output power) 
A NC program generated by the CAMAM is executed in the $\mathrm{NC}$ of the based machine and commands for changing the variable are loaded and transmitted to each controller, then these variables become in effect. It should be noted that while motion commands are transmitted directly from the $\mathrm{NC}$ to the motion controller, powder feeder controller and laser power controller are controlled by programmable logic controller (PLC). This difference generates response characteristics.

\section{Optimization of workpiece orientation}

\subsection{Discussion of workpiece orientation effect on deposition condition}

From the viewpoint of the process principle described in chapter 1, successful deposition requires consistent formation of a melt pool on the surface of a work piece. Main considerations for maintaining a melt pool size and shape are energy input and material input. The orientation of the workpiece surface relative to the deposition nozzle which is fixed in gravity direction can influence both of these things. Therefore, to maintain a consistent melt pool, the workpiece surface must maintain the same orientation relative to the laser beam as well as be provided with sufficient energy to form a melt pool of a specific size. Due to the variability and dynamic nature of processing and environmental conditions in DED, it is difficult to predict required changes in laser energy output needed to maintain a melt pool of constant size. Such challenges are better solved using real time monitoring and feedback control. Alternatively, the workpiece orientation is defined in the NC program and needs to be taken into account by the CAM system during additive path generation. It has been observed that tilting the workpiece surface to get its surface normal vector be away from the gravity direction can change the shape of the laser spot projected onto a planer surface (Lin et al., 1999). The altered spot shape results in non-uniform energy density distribution that affects the melt pool shape. It is apparent for the case of planer and spherical surfaces that the deposition surface which is perpendicular to the nozzle direction minimizes deformations in the laser applied area. From this, it is perceived that formation of the uniformly shaped melt pool requires that the workpiece orientation be set such that its surface normal vector can be directed to the nozzle. Figure 4 illustrates above discussion by demonstrating the deformation in laser applied area and non-uniform distribution of powder across the workpiece, when the surface normal vector is aligned offset from the nozzle direction for an arbitrary surface.

\subsection{Optimization of workpiece orientation}

According to the above discussion, what it needs to pay attention to regarding deposition of especially 3D shapes on the free form surface is controlling the workpiece orientation against the direction of the laser nozzle fixed to the gravity direction by using simultaneous five-axis motions. It should be noted that the workpiece orientation which is dictated by the base CAM does not always have its surface normal vector be the same as the nozzle direction and thus not optimized for the AM process. In this study, the workpiece orientation at each tool path point has been modified such that the surface normal vector which is evaluated as below can coincide with the nozzle direction. First, against a target tool path curve, an offset curve with small spacing to the target curve is evaluated along the mid-surface of the shape as shown in Fig.5. Second, an equal number of points with uniform spacing are evaluated along both curves and these points on the target curve will become tool path points. Third, a vector between each tool path point and its corresponding point on the next layer path curve is evaluated as $\vec{N}$. Next, the tangent to the tool path curve is calculated at each tool path point as $\vec{T}$. Then, a normal vector to blade side surface, $\vec{V}$ is evaluated at each tool path point as cross product of $\vec{T}$ and $\vec{N}(\vec{V}=$ $\vec{T} \times \vec{N})$. Finally, the cross product is repeated with the path tangent and the side surface normal vectors to get the desired surface normal vector, $\overrightarrow{N^{\prime}}=\vec{V} \times \vec{T}$.

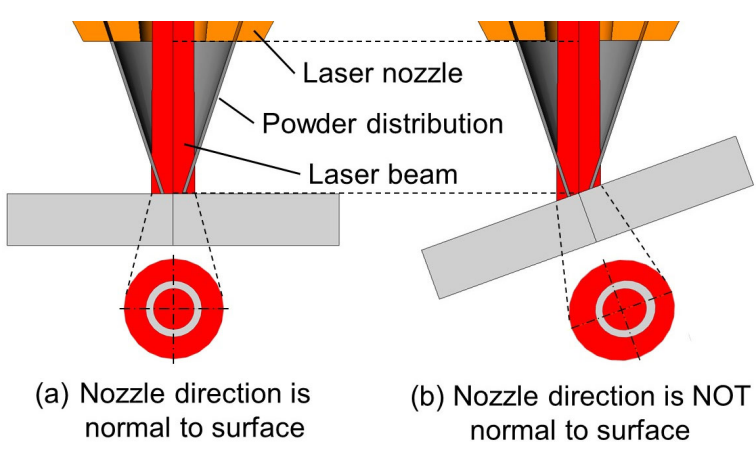

Fig. 4 Schematic of workpiece orientation effect on laser applied area shape and powder distribution.

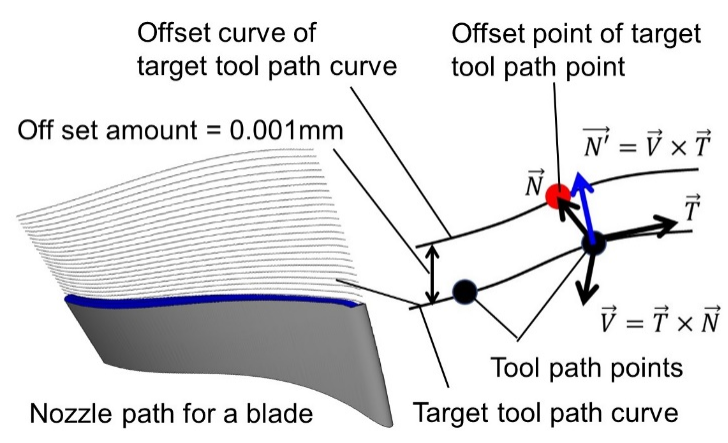

Fig.5 Evaluation of surface normal vector at tool path point. 


\section{Conceptual model of 3D CAM system for DED type additive manufacturing process}

According to past research, typical path planning methods for the AM process used mostly 2D or 3D slicing method (Urbanic et al., 2017). In the conventional method, the laser application path was generated by establishing a cross section with $2 \mathrm{D}$ cut planes of the various orientations to the $3 \mathrm{D}$ geometry of the part. However, the DED process requires more generative path planning method to deposit material efficiently by making the best use of its unique feature of 3D deposition according to the optimal orientation for the feature to be built. In the study, a new method has been proposed for creating versatile 3D deposition paths which control both the position of the nozzle to the work piece and process parameters along the path. The concept shown in Fig. 6 relies on the idea that the 3D deposition path is created by reversal playback of the subtractive machining path which removes the volume of the targeted part geometry until it has null geometry that means no volume. Such subtractive machining path can be calculated by using the tool path generation algorithms available in the commercial CAM software for cutting operations. In addition, the instantaneous material removal volume is recorded for every path segment that is the unit length dividing the subtractive machining path, as the cutting tool moves to remove the portion of the product. Regarding the calculation of the material removal volume at each path segment, the virtual simulation integrated in the base CAM is used and the algorithm was originally studied in (Wang et al., 1988). In this study, an end mill type cutting tool showed in Fig. 7 is used for the virtual simulation. It should be noted that the blade portion of the targeted part showed in Fig. 6 has been removed without remaining in the width direction only by single line paths which pass through the center of the blade in the width direction because the thickness of the blade portion is the same as the diameter of the cutting tool and the subtractive machining path is a trajectory of the center of the cutting tool. While such subtractive machining path might not be used for general cutting, DED paths need to pass the center of the targeted part in the width direction since the material has been deposited in both side of the paths. After storing the all paths with the material removal volume recorded at each path segment, the information is reversely replayed each by each from the last path segment toward the first path segment such that the 3D targeted shape can be built from scratch. The recorded removal volume is equated to the volume required to produce the desired object during the deposition process. With the volume and corresponding position data, the process model constructed based on empirical data as described in chapter 5 is used to evaluate the process parameters required to achieve the desired volume along the deposition path. Above description is formulated as below;

$$
\begin{aligned}
& V_{n}=\varphi_{n_{(X)}} \\
& X=\varphi_{n}{ }^{-1}\left(V_{n}\right)
\end{aligned}
$$

Here $V_{n}$ is the material removal volume calculated by the virtual simulation for $\mathrm{n}^{\text {th }}$ path segment, $\varphi_{n}$ is the process model-based function described in chapter 5 and $X$ is a process parameter which can be represented by $Q_{m}, P$ or $F$. Figure 7 shows the simulation of the in-process part geometry using solid modelling (Wang et al., 1988). In this method, the work piece and the swept volume of the tool paths are modelled as solids, the machined surface can be generated by taking a Boolean difference between these volumes. Then the difference between stock volumes of the work piece before and after machining by the $\mathrm{n}^{\text {th }}$ path segment is calculated as the material removal volume for the path segment.

$$
V_{n}=V o l_{n}-\operatorname{Vol}_{n-1}
$$

Figure 8 is an example of material removal volume per path segment which is recorded in the subtractive process of the blade shown in Fig.6(a). With regard to oscillating fluctuations which can be seen in the figure, these are caused since the direction of the all paths is from the lower side of the blade to the higher side for each layer and the distance between a path and its adjoining path is changing along the path. It should be noted that the nozzle orientation needs to be optimized as discussed in chapter 3 before performing the subtractive simulation for material removal volume. Figure 9 is the reversed order of Fig. 8 that means deposition volume per path segment in the DED process of the blade. Also, the process parameter, in this case the nozzle feed rate, which is evaluated from the volume by using the process modelbased function is illustrated in the figure. When the subtractive path is reversed, its last path segment corresponds with the first path segment of the DED path. In this example, for subtractive machining path generation, a cutting tool which diameter is $3 \mathrm{~mm}$ that is the diameter of the laser spot used during the experiment was used and path segment length was set to be $1 \mathrm{~mm}$ throughout the path. The total number of path segments is 1,697. It should be noted that some path segments of the subtractive machining path are for approach or evacuation motion where the removal volume is 
calculated as zero. For such path segments, process parameters should be exceptionally determined and in this case the nozzle feed rate was uniformity set to $1,000 \mathrm{~mm} / \mathrm{min}$ that is a normal value of rapid feed rate for this system. The reversed DED path and the evaluated process parameters integrated into the path data were finally converted using a post processor into a functional NC program. Figure 10 illustrates the successive flow of generating NC programs for DED discussed above. The idea is very simple and feasible for creating NC programs for DED type additive manufacturing by integrating custom control functions for process elements such as powder supply, laser power and nozzle motion into a conventional 3D CAM system, which can generate 3D machining programs with 3D material removal simulation. The concept mentioned above is for the unique 3D CAM system for DED type additive manufacturing to be developed as CAMAM.

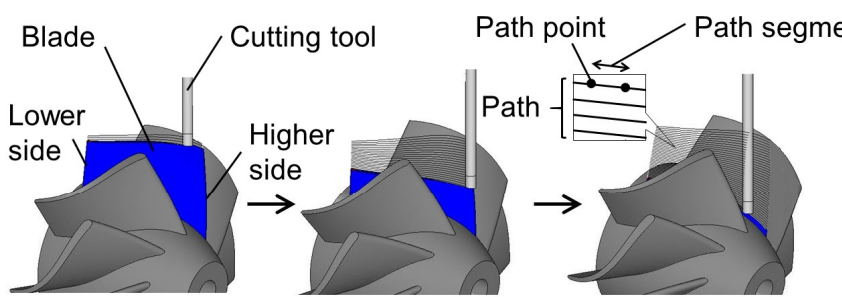

(a) Subtractive machining path which removes entire volume of the blade portion

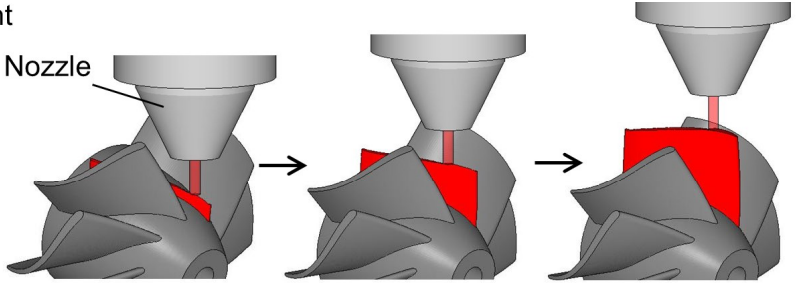

(b) DED path (reversal play of (a))

Fig. 6 Example of proposed method to create 3D deposition path of the blade portion.

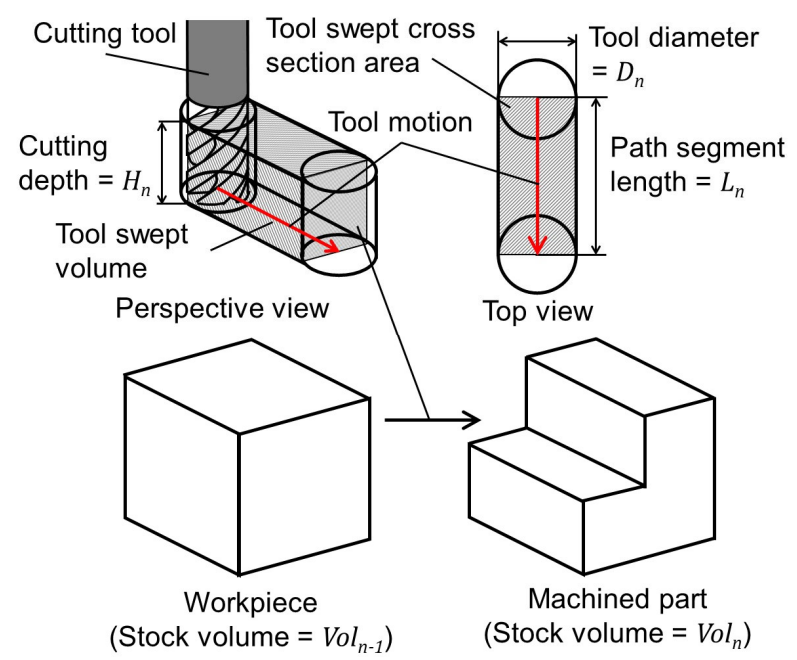

Fig. 7 Schematic of spatial domain swept by cutting tool during one path segment motion.

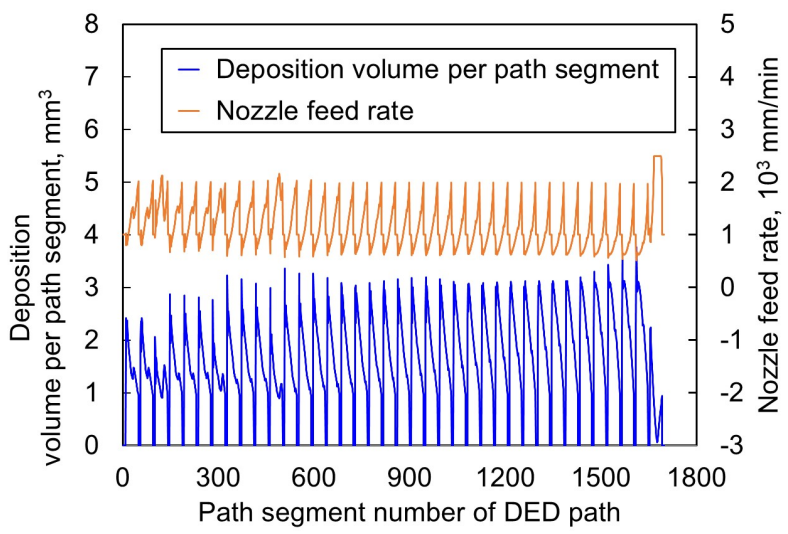

Fig. 9 Deposition volume per path segment in DED portion (reversal replay of Fig.8).

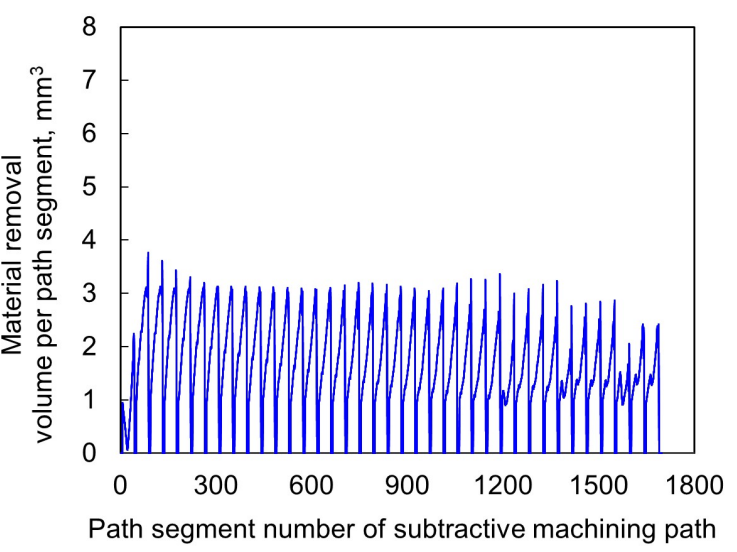

Fig. 8 Material removal volume per path segment recorded in subtractive machining portion in case of blade part shown in Fig.6 (a).

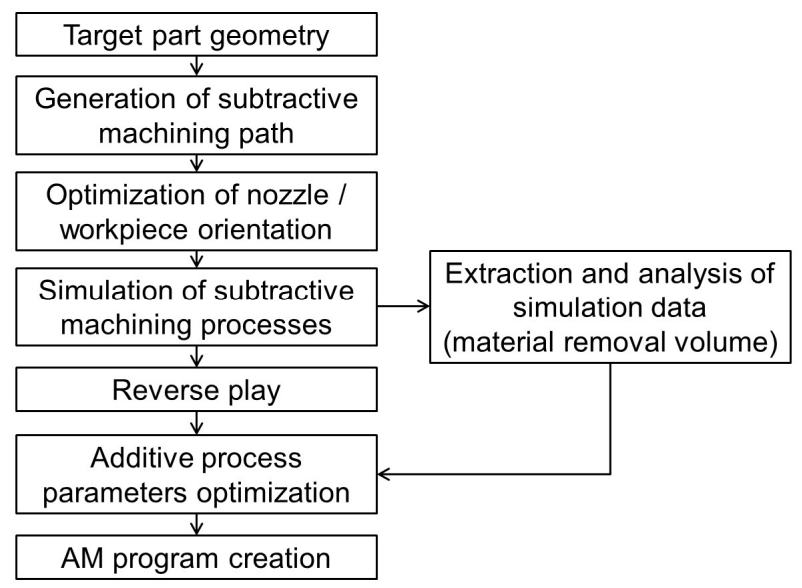

Fig. 10 Flow of generating NC program for DED using CAMAM. 


\section{Deposition process parameter models}

\subsection{Fundamental test to dictate deposition process parameter models}

To study the relationships between the process parameters and the deposition volume to obtain the process modelbased function described in the previous chapter, single lines were deposited by dynamically changing the process parameters along the lines. In the study, stainless steel powder 316L powder (particle size distribution ranges: 45-106 $\mu \mathrm{m}$ ) and D2 tool steel as a base plate were used. Argon gas was used as shield and powder carrying gas. After each section of 5 paths, the powder mass flow rate, $Q_{m}$ was changed from 7.7, 14.0, 17.1, 20.3, 26.6 to $32.9 \mathrm{~g} / \mathrm{min}$. Within 5 lines, the laser power, $P$ was changed from 800 to 2000 by increments of $300 \mathrm{~W}$. For every line, the nozzle feed rate, $F$ was changed from 500 to 2500 by increments of $500 \mathrm{~mm} / \mathrm{min}$. Figure 11 shows a picture of deposited single lines. The geometry of deposited lines was measured using a coordinate measuring machine (CMM). After the measurement, statistical analysis of the relationship between the geometric characteristic of the deposition was completed using the combined parameter $P^{\alpha} Q_{m}{ }^{\beta} F^{\gamma}$ proposed in (Onwubolu et al., 2007), (Cheikh et al., 2012). Coefficients for $\alpha$, $\beta$ and $\gamma$ were evaluated using linear regression of the log function. Finally, a relationship as $y=a\left(P^{\alpha} Q_{m}{ }^{\beta} F^{\gamma}\right)+b$ was obtained, where $y$ represents a geometric parameter of the deposition while $a$ and $b$ are the coefficients of the best fit line. With experimental results obtained in this study, it was found that the deposition height ( $=H[\mathrm{~mm}])$ is proportional to $Q_{m} / F$ parameter with a correlation coefficient $R^{2}=0.85$ considering all the 105 experiments. Figure 12 shows this result. It is worth noting that the laser power does not show up in the combined parameter. This indicates that the effects of the laser power on the deposition height are relatively small and agrees with results in (Oliveira et al., 2005). It should be noted that in this study a linear regression model was used for simplicity while higher-order regression model might be applied for more fitting to experimental data. If such model is used, the same procedure needs to be taken to determine the relationship between the nozzle feed rate and deposition volume that is discussed in the next section. In the experiments, it was also visually confirmed that no deposition was occurred at $P$ of $800 \mathrm{~W}$ and the deposited quality deteriorated at $Q_{m}$ of $7.7 \mathrm{~g} / \mathrm{min}$ or $F$ of $2500 \mathrm{~mm} / \mathrm{min}$. These experiments were excluded from Fig. 12. Based on these facts, it is assumed that the relationship can come into effect as far as the process parameters are better than above values. It should be noted that the same procedure needs to be taken to determine the relationships for calibration in a case of using different materials.

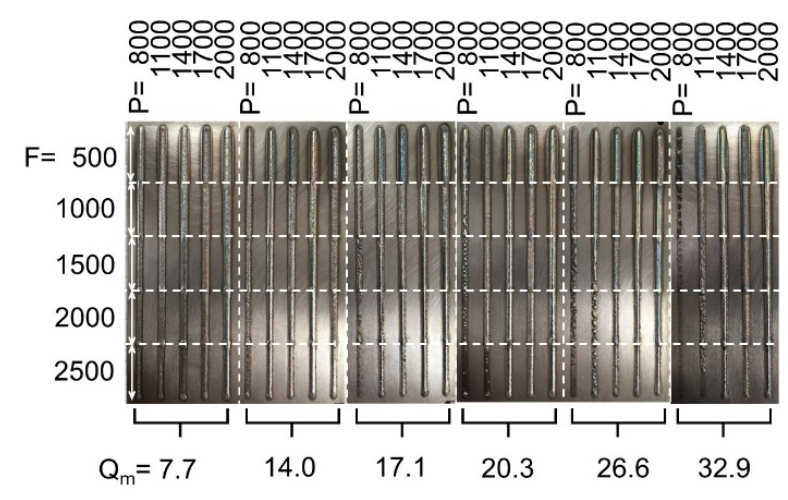

Fig.11 Picture of deposited single lines for fundamental tests.

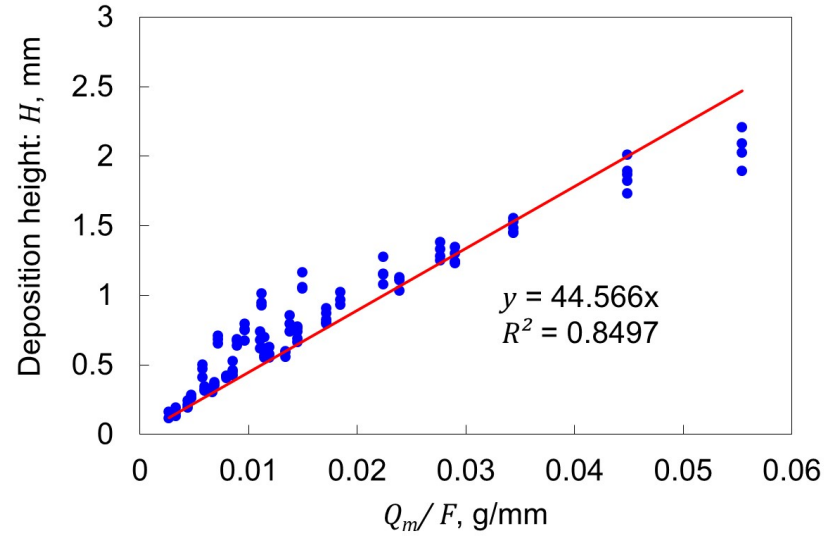

Fig. 12 Deposition height as a function of combined parameter $Q_{m} / F$.

\subsection{Deposition volume control based on nozzle feed rate}

As a result of the subtractive simulation, which is performed by the base CAM software, there is the variation of the material removal volume per path segment for each path segment as shown on Fig.8. When the path is reversely replayed for the deposition, the material removal volume per path segment is changed to be the deposition volume per path segment. Here, it should be noted that the supplied volume per path segment, $V_{d}$ can be described by the following equation;

$$
V_{d}=Q_{m} / F
$$

In order to vary this value, $Q_{m}$ is supposed to be changed while $F$ might stay in constant because $Q_{m}$ can affect directly the deposition volume per path segment. On the other hand, the response time of powder supply is relatively 
long (Ueda et al., 2018). Figure 13 shows the result of experiments of dynamic powder weight measurement when the $\mathrm{NC}$ commands of changing the powder mass flow rate in a step wise manner were executed. Here, the dynamic change of the powder weight was monitored when the powder was being fed into the bottle, which was placed on the digital scale. As shown in Fig. 13, it was identified that the powder mass flow rate control has about 15 seconds response time delay for the actual powder mass flow rate to reach the targeted value after NC commands are given to the CNC controller. Regarding the response delay time of the nozzle feed rate, it was estimated by using a built-in NC controller recording function of axes velocity. As the result, it was monitored that it took 0.04 second to accelerate from 0 to $1,000 \mathrm{~mm} / \mathrm{min}$ under the sampling frequency of 0.004 second. From these results, although the evaluated response delay time of the nozzle feed rate should depend on the amount of change, it is negligible compared to that of other parameters when designing additive operations.

Since powder supply has such response time delay, in this study, $F$ was changed to dynamically adjust the deposition volume along the path as the more practical way while keeping $Q_{m}$ constant $(14.5 \mathrm{~g} / \mathrm{min})$. If it needs to adjust $Q_{m}$ to change the deposition volume, it is necessary to wait until the powder mass flow rate achieves the targeted value by introducing dwell time. In addition, even though $F$ has some effects on the deposition width, it is small relative to the change in deposition height and for simplicity assumed constant at the nozzle feed rate of typical operations. In this study, based on these approximations, the material removal volume calculated by the virtual simulation was approximated by the following equations;

$$
\begin{aligned}
& V_{n}=\varphi_{n_{(X)}}=D_{n} \cdot L_{n} \cdot H_{n} \\
& H_{n}=6.46 \times 10^{2} \cdot F_{n}{ }^{-1}
\end{aligned}
$$

Here $L_{n}$ is the path segment length, $D_{n}$ is the tool diameter and $H_{n}$ is the cutting depth at $\mathrm{n}^{\text {th }}$ path segment as shown in Fig.7. Equation (6) has been led by the relationship expressed in Fig.12 under the condition that the powder mass flow rate is constant. In this study, it has been assumed that the same cutting tool is used for entire subtractive machining process even though a cutting tool might have been sometimes changed during the actual cutting process. Under such assumption, $D_{n}$ is kept being constant during the virtual simulation. In addition, $D_{n}$ is equivalent to the deposition width of the deposition process. Here, it should be noted that deposition width differs according to the laser power as shown in Fig.11. For the above assumption, the laser power has been set to the constant value of $1700 \mathrm{~W}$ and the deposition width at this power $(=3 \mathrm{~mm})$ has been used as the tool diameter for the virtual simulation. Conclusively, the deposition volume per $1 \mathrm{~mm}$ path segment length varies according to the nozzle feed rate change as expressed by the following equation;

$$
F_{n}=\varphi_{n}{ }_{\left(V_{n}\right)}^{-1}=1.94 \times 10^{3} \cdot V_{n}^{-1}
$$

Figure 14 shows this equation graphically. According to this relationship, the nozzle feed rate, $F_{n}$ was calculated for each path segment from deposited volume determined by the volume simulation described in chapter 4 .

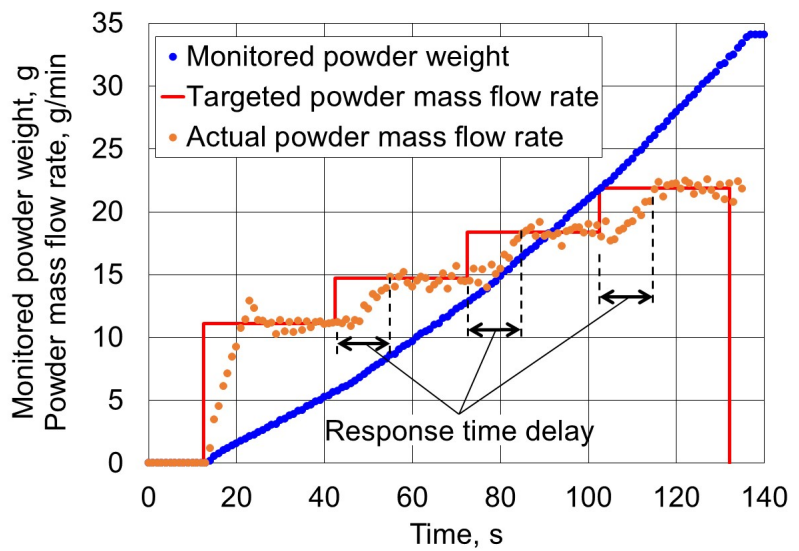

Fig. 13 Powder mass flow rate change against NC commands (carrier gas flow rate is $6 \mathrm{~L} / \mathrm{min}$ ).

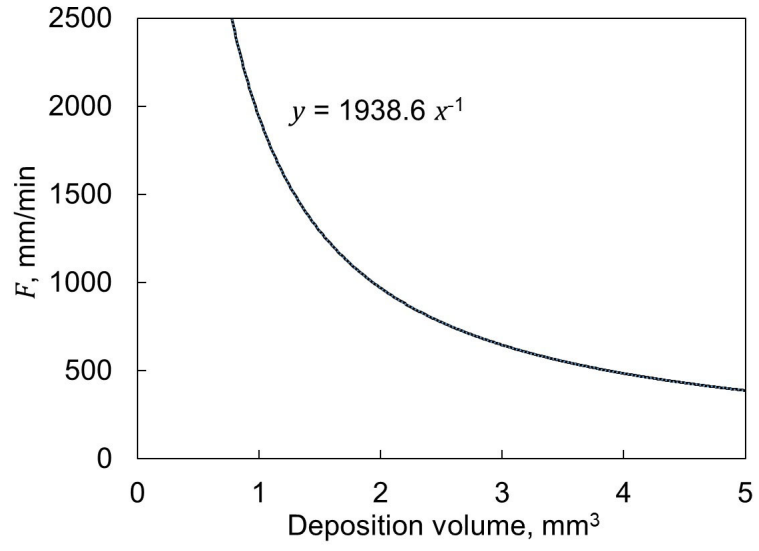

Fig. 14 Nozzle feed rate, $F$ as a function of deposition volume (path segment length is $1 \mathrm{~mm}, Q_{m}=14.5 \mathrm{~g}$ ). 


\section{Verification of the developed CAM system feasibility \\ 6.1 Verification of $\mathrm{NC}$ program based volumetric deposition rate control}

For evaluating the effectiveness of the developed CAM system, firstly the single line with piecewise different height shown in Fig. 15(a) was designed. The geometry of the line is shown as 'Model height' (blue line) in Fig.15(c) and consist of 1 layer. The subtractive machining path was generated by discretizing the line with $1 \mathrm{~mm}$ in length. After definition of the path geometry, the material removal simulation was performed, and its data was recorded for each discretized element which is shown as 'deposition volume' (green dots) in Fig.15(c). The simulation was followed by approximation of the deposition height from the tool geometry which diameter was same as the width of the model and the volumetric data along the path. The approximated deposition thickness was then used to evaluate the required nozzle feed rate to achieve the desired value from the empirical relationships evaluated in chapter 5. Orange dots in Fig.15(c) indicated such evaluated nozzle feed rate at each tool path point. Finally, using the feed rate, the single line was deposited. Here, the laser power and the powder mass flow rate were kept being constant to $1,700 \mathrm{~W}$ and $14.5 \mathrm{~g} / \mathrm{min}$ for the entire process. Figure 15(b) displays a picture of the deposited line. After the deposition, the geometry of the line was evaluated by taking measurements of the cross-sectional profile and 'Measured height', red dots in Fig.15(c), were obtained. A comparison of the measurement and the original solid model is shown in Fig. 15(c). As seen in the figure, it has been concluded that the geometry deposited had a good agreement of the target geometry with good accuracy.

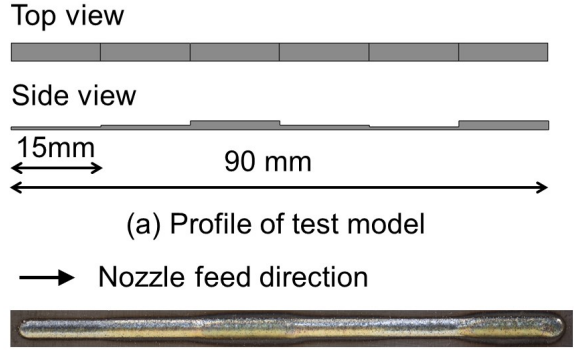

(b) Picture of deposited part (Top view)

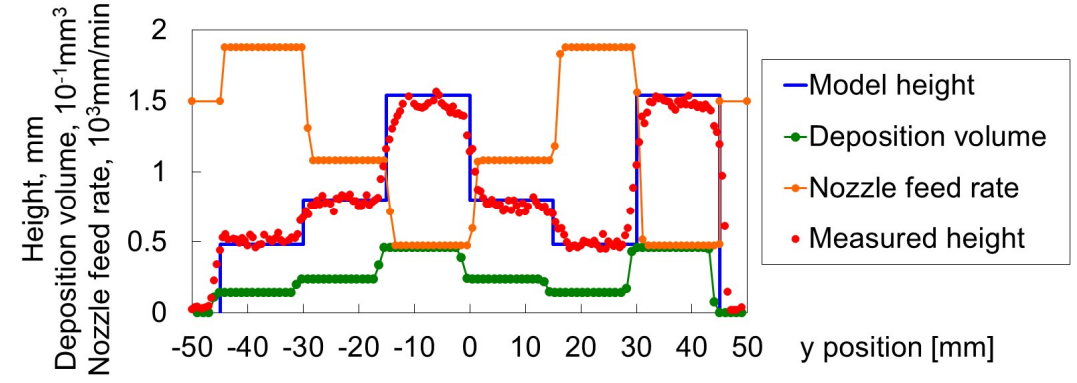

(c) Measured height compared to model geometry

Fig. 15 Deposition of the single line.

\subsection{Deposition of two-dimensional object}

The applicability of the CAMAM to fabricate the two-dimensional geometry was also evaluated by creating the geometry, whose solid model is shown in Fig. 16(a). Deposition of such thin-walled objects, in which the top surface is not parallel to the bottom surface may result in the distorted shape if traditional horizontally-slicing method is applied (Pandey et al., 2003). In the prototyped CAMAM system, non-uniform isoperimetric slicing method which is equipped in the commercial CAM software as a geometric processing method has been utilized. The algorithm was studied in the computer graphics field (Salomon, 2006). In order to implement this method for such non-uniform 2D geometry deposition, firstly, the middle surface between the both side surfaces of the model is created in the commercial CAM software as a parametric surface which has two parametric directions illustrated in Fig. 16(b) as $u$ and $v$. Then, by means of the slicing method, a surface curve is created on a surface along $v$ direction of the middle surface. By repeating the creation of surface curves so as to divide the middle surface equally in $u$ direction, inclined layered curves evenly spaced with variable pitch are generated. In the example shown in Fig. 16(c), the height was automatically divided into 57 layers and the deposition path was generated by connecting the lines. After that, the same steps as explained for the single line deposition in section 6.1 were taken to generate the NC program. One different point is the relationship between the deposition volume and the required nozzle feed rate. In the multiple layers deposition, from prior experiments, it was confirmed that the relationship need to be changed between 1st layer and the other. Figure 17 shows the nozzle feed rate modulation along the tool path for each layer. It should be noted that the laser power and the powder mass flow rate were kept being constant to $1,700 \mathrm{~W}$ and $14.5 \mathrm{~g} / \mathrm{min}$ during the process. The deposition result shown in Fig. 16(d) shows the success of such adaptive layer stacking method resulting in the coincidence of the deposited object with the solid model designed. No distortion of the deposited geometry has been observed. It should be noted that in the above case the deposition height for each layer gradually change and its variation is not so much and can be complemented with modulation of the nozzle feed rate. On the other hand, some targeted parts might have a very large height variation in its geometry that is not able to be complemented even if the nozzle feed rate is zero because the 
deposition height is not infinite. In such case, to divide the part could be one solution. This means that it is necessary to split the part into sub-shape which has an acceptable variation of deposition height, create the deposition path for each shape and perform the deposition processes one by one.

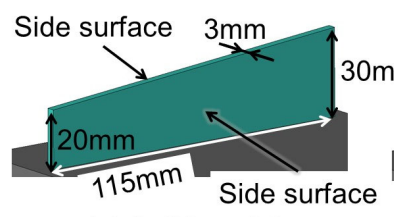

(a) Solid model

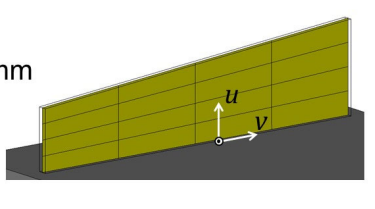

(b) Middle surface

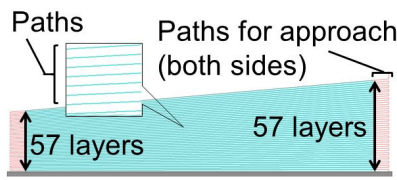

(c) Deposition path

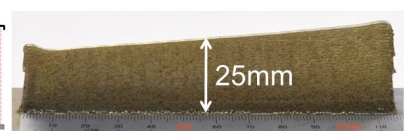

(d) Deposited part

Fig. 16 Deposition of the sloping thin wall.

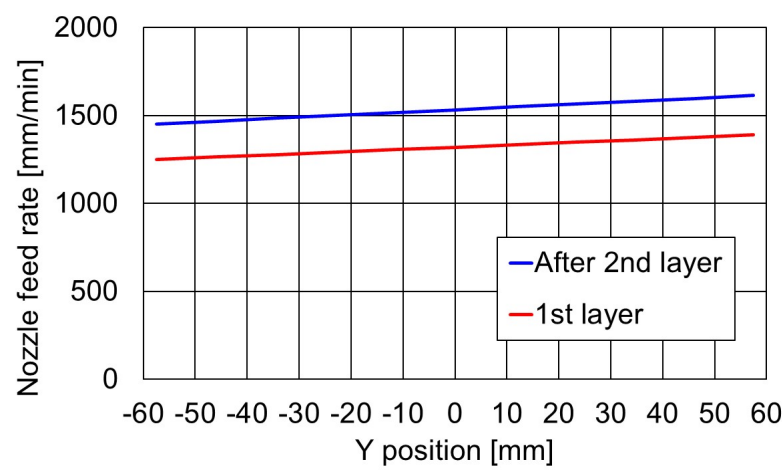

Fig.17 Nozzle feed rate modulation along tool path.

\subsection{Fabrication of three-dimensional shaped part}

In the previous sections, the feasibility of the developed CAM has been demonstrated using one and two-dimensional parts. In this section, the proposed path generation method has been applied to create more complex three-dimensional part. Figure 18(a) shows a 3D solid model of the target three-dimensional object which is a part similar to a blisk. To perform the DED process to create this shape, the part was isolated into the core portion and blades as shown in Fig. 18(b) and (c). Using traditional spiral paths of subtractive machining generated by using the base CAM, the DED paths for the core were reversely regenerated as shown from Fig.18(d). For the blades, the isoperimetric path generation method explained in section 6.2, were used to build the uneven and free form blade structures as shown from Fig.18(e). Figure 19(a) shows a picture of the part from which successful deposition by using proposed path generation method has been confirmed. At the same time, there is still the remaining challenge that the mechanical properties and microstructures that define the quality of deposited parts have to be evaluated. As energy input per unit time and cooling rate of deposited part can be affected by nozzle feed rate change, such characteristics can be affected as well (Amine et al., 2014), (Hansel et al., 2016). Therefore, in a future, it would be required to clarify the range of the nozzle feed rate that can be accepted from those viewpoints, and to plan the deposition height and path so that the nozzle feed rate can change in the range.

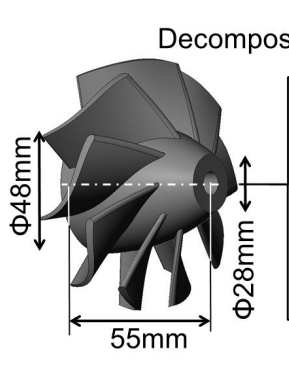

(a) 3D solid model

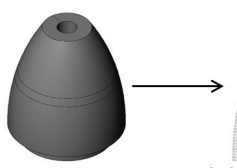

(b) Core portion
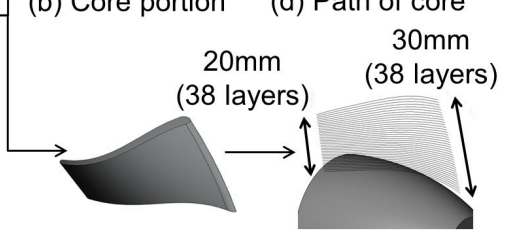

(c) Blade portion

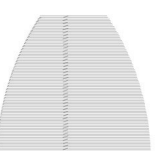

(d) Path of core $30 \mathrm{~mm}$ (38 layers)

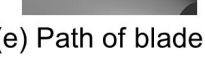

Fig. 18 Deposition path for each feature of the part.

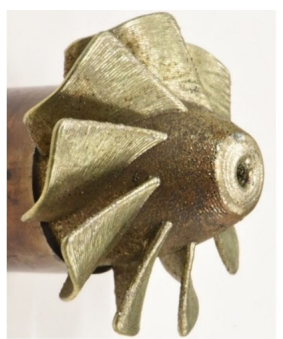

(a) Geometrically accurate part (Workpiece orientation optimized)

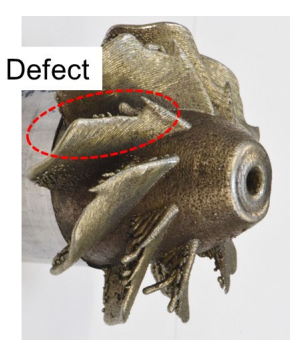

(b) Defective part

(Workpiece orientation NOT optimized)
Fig. 19 Pictures of the deposited part.

\subsection{Efficacy of workpiece orientation optimization}

In order to verify the efficacy of workpiece orientation optimization described in chapter 3 , another part has been deposited with different conditions of the workpiece orientation resulting in defect occurred during blade deposition as shown in Fig.19(b). In case of the deposition shown in Fig. 19(b), the workpiece orientation was set such that the surface normal vector of the core portion coincides with the nozzle direction and was kept being constant throughout whole layers. It should be noted that the surface normal vector described in this section always means the outer direction of the model or the surface to be deposited. According to increase of the number of layers, the angle between the nozzle direction and the surface to be deposited was getting away from normal, and then it finally resulted on the defect shown in the figure. On the other hand, during the deposition of the blades shown in Fig. 19(a), the workpiece orientation was 
continuously being changed according to layer increment. Until the blades grew to the beginning third portion, the workpiece was orientated such that the resultant vector mixed with the surface normal vector of the core portion and that of the blade cross section can coincide with the nozzle direction. After the beginning third portion, the surface normal vector of the blade cross section had been being the same as the nozzle direction. Controlling the workpiece orientation in this manner, previously deposited layers remained normal to the nozzle which is fixed to the gravity direction and flaws of the blades resulting from improper orientation were avoided. To evaluate the deviation of the surface normal vector of the deposited layer from the nozzle direction, the following value, $D$ was calculated for each tool path point as the magnitude of the deviation.

$$
D=\sqrt{\left(N_{x}^{\prime}-V_{x}^{\prime}\right)^{2}+\left(N^{\prime}{ }_{y}-V_{y}^{\prime}\right)^{2}+\left(N_{z}^{\prime}-V_{z}^{\prime}\right)^{2}}
$$

Here $\overrightarrow{N^{\prime}}=\left(N_{x}^{\prime}, N_{y}^{\prime}, N_{z}^{\prime}\right)$ is the surface normal vector and $\overrightarrow{V^{\prime}}=\left(V_{x}^{\prime}, V_{y}^{\prime}, V_{z}^{\prime}\right)$ is the nozzle direction vector at each tool path point. Both vectors are the unit vector. It should be noted that the nozzle direction vector turns out to be $(0,0,-1)$ since the nozzle is fixed to the gravity direction in the system used in this study. In Fig. 20, the calculated deviation is mapped in three-dimensions in addition to displaying pictures of both geometrically accurate and defective parts. In Fig. 20, the magnitude of the deviation shows a comparably higher value in lower part of the defective blade. It can be assumed that deposition failure was led in subsequent layers of the defective blade due to growing deviations originating from the lower layers and worsening of melt pool formation.

These results imply that stable melt pool formation has a close relationship with the stability of the deposition process. Controlling the workpiece orientation to match the surface normal vector of the deposited layer with the nozzle direction throughout the deposition process allows for the possibility of stable melt pool formation with sufficient size for deposition at the targeted position on the pre-deposited surface, resulting in a stable deposition process.

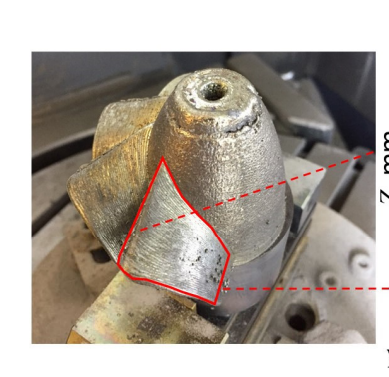

(a) Geometrically accurate blade

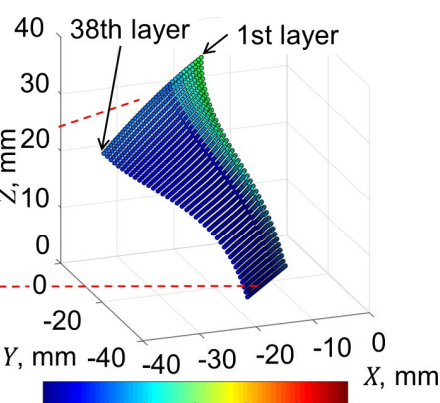

$\begin{array}{lllllll}0 & 0.2 & 0.4 & 0.6 & 0.8 & 1.0 & 1.2\end{array}$

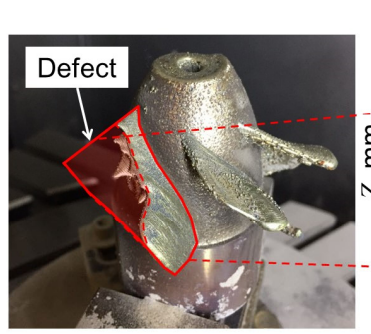

(b) Defective blade

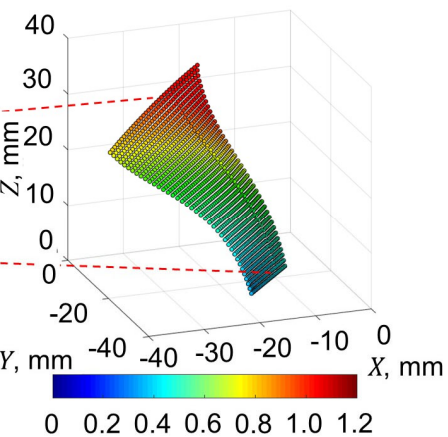

$\begin{array}{llllllll}0 & 0.2 & 0.4 & 0.6 & 0.8 & 1.0 & 1.2\end{array}$

Fig. 20 Pictures of deposited blade and 3D mapping of magnitude of deviation, $D$ at each tool path point.

\section{Conclusions}

Aiming at successful and efficient operations of DED type AM system, the dedicated CAM system referred to as "CAMAM" has been proposed with the focus on autonomous deposition optimization. The results in this study lead to the following conclusions.

(1). DED path generation methodology was proposed as reverse playing back of the subtractive machining paths for machining whole object to the null.

(2). Adaptive deposition process control was proposed and developed based on the evaluation of the relationship between the process parameters and the deposition volume.

(3). Based on above proposed approach, the dedicated CAM system was developed, and its feasibility was verified by fabricating of one-, two-, and three- dimensional part models that are limited to shapes created by one-line deposition.

(4). From the results of three-dimensional part deposition, it can be suggested that by controlling the workpiece orientation to match the surface normal vector of the pre-deposited surface with the direction of the nozzle fixed to the gravity direction throughout the process, laser applied area shape and powder distribution kept being uniform, and the melt pool can be stably formed at the targeted position on the surface, thus the deposition process itself become stable. 


\section{References}

Amine, T., Newkirk, JW., Liou, Fran., An investigation of the effect of direct metal deposition parameters on the characteristics of the deposited layers, Case Studies in Thermal Engineering, Vol. 3 (2014), pp.21-34.

Autodesk Inc., Autodesk introduces PowerMill Additive for high rate additive manufacturing, $<$ https://knowledge.autodesk.com/support/powermill/getting-started/caas/CloudHelp/cloudhelp/2019/ENU/PWR M-Additive/files/GUID-5B32F5CB-8DDC-475C-9450-07285615BF9E-htm.html>, (2019).

CGTech Ltd., ADDITIVE VERICUT Module, <https://www.cgtech.com/products/about-vericut/additive/>, (2018)

Cheikh, HE., Courant, B., Branchu, S., Hascoet, JY., Guillen, R., Analysis and prediction of single laser tracks geometrical characteristics in coaxial laser cladding process, Optics and Lasers in Engineering, Vol.50 (2012), pp.413-422.

Ding, Y., Warton, J., Kovacevic, R., Development of sensing and control system for robotized laser-based direct metal addition system, Additive Manufacturing, Vol.10 (2016), pp.24-35.

Hansel, A., Mori, M., Fujishima, M., Oda, Y., Hyatt, G., Laverni, E., Delplanque, JP., Study on consistently optimum deposition conditions of typical metal material using additive/subtractive hybrid machine tool, Procedia CIRP, Vol. 46 (2016), pp.579- 582.

Kuriya, T., Koike, R., Mori, T. and Kakinuma, Y., Relationship between solidification time and porosity with directed energy deposition of Inconel 718, Journal of Advanced Mechanical Design, Systems, and Manufacturing, Vol. 12, No. 5 (2018), DOI: 10.1299/jamdsm.2018jamdsm0104.

Lin, J. and Hwang, B. C., Coaxial laser cladding on an inclined substrate, Opt. Laser Technol., Vol.31, No.8 (1999), pp.571-578.

Liou, F., Ruan, J., Sparks, TE., Multi-Axis Planning System (MAPS) for Hybrid Laser Metal Deposition Processes, Department of Mechanical and Aerospace Engineering Missouri University of Science and Technology, (2010), pp.592-605.

Liu, Y., Yang, YQ., Wang, D., A study on the residual stress during selective laser melting (SLM) of metallic powder, The International Journal of Advanced Manufacturing Technology, Vol.87 (2016), pp.647-656.

Newman, ST., Zhu, Z., Dhokia, V., Shokrani, A., Process Planning for Additive and Subtractive Manufacturing Technologies, CIRP Annals-Manufacturing Technology, Vol.64 (2015), pp.467-470.

Oliveira., U., Ocelı'k, V., Hosson, J.Th.M., Analysis of coaxial laser cladding processing conditions, Surface \& Coatings Technology, Vol.197 (2005), pp.127-136.

Onwubolu, C., Davim, PJ., Oliveira, C., Cardoso, A., Prediction of clad angle in laser cladding by powder using response surface methodology and scatter search, Optics \& Laser Technology, Vol.39 (2007), pp.1130-1134.

Pandey, PM., Reddy, NV., Dhande, SG., Slicing procedures in layered manufacturing: a review, Rapid Prototyping Journal, Vol.9, No.5 (2003), pp.274-288.

Salomon, D., Curves and Surfaces for Computer Graphics, Springer, ISBN 978-0-387-28452-1 (2006)

Schmidt, M., Merklein, M., Bourell, D., Dimitrov, D., Hausotte, T., Wegener, K., Overmeyer, L., Vollertsen, F., Levy, G., Laser based additive manufacturing in industry and academia, CIRP Annals-Manufacturing Technology, Vol.66 (2017), pp.561-583.

Thompson, MK., Moroni, G., Vaneker, T., Fadel, G., Campbel, R., Gibson, I., Bernard, A., Schulz, J., Graf, P., Ahuja, B., Martina, F., Design for Additive Manufacturing: Trends, Opportunities, Considerations, and Constraints, CIRP Annals_-Manufacturing Technology, Vol.65 (2016), pp.737-760.

Ueda, M., Carter, D., Yamazaki, K., Kakinuma, Y., Identification of Dynamic Process Response in DED-type Additive Manufacturing Process and Its Application to Process Optimization, Proceedings of 17th International Conference on Precision Engineering (ICPE 2018) (2018), Paper No. C000052.

Urbanic, RJ., Hedrick, RW., Burford, CG., A process planning framework and virtual representation for bead-based additive manufacturing processes, The International Journal of Advanced Manufacturing Technology, Vol.90 (2017), pp.361-376.

Wang, W.P., Solid Modeling for Optimizing Metal Removal of Three-dimensional NC End Milling, Journal of Manufacturing System, Vol.7, No.1 (1988), pp.57-65.

Xiong, J., Zhang, G., Qiu, Z., Li, Y., Vision-sensing and bead width control of a single-bead multi-layer part: material and energy savings in GMAW-based rapid manufacturing, Journal of Cleaner Production, Vol.41 (2013), pp.82-88. 